\title{
Hyperthyroidism in adolescents
}

\author{
Marek Niedziela
}

Department of Pediatric Endocrinology and Rheumatology, Institute of Pediatrics, Karol Jonscher's Clinical Hospital, Poznan University of Medical

Sciences, Poznan, Poland

Correspondence should be addressed to M Niedziela: mniedzie@ump.edu.pl

\begin{abstract}
The term 'hyperthyroidism' refers to a form of thyrotoxicosis due to inappropriate high synthesis and secretion of thyroid hormone(s) by the thyroid. The leading cause of hyperthyroidism in adolescents is Graves' disease (GD); however, one should also consider other potential causes, such as toxic nodular goitre (single or multinodular), and other rare disorders leading to excessive production and release of thyroid hormones. The term 'thyrotoxicosis' refers to a clinical state resulting from inappropriate high thyroid hormone action in tissues, generally due to inappropriate high tissue thyroid hormone levels. Thyrotoxicosis is a condition with multiple aetiologies, manifestations, and potential modes of therapy. By definition, the extrathyroidal sources of excessive amounts of thyroid hormones, such as iatrogenic thyrotoxicosis, factitious ingestion of thyroid hormone, or struma ovarii, do not include hyperthyroidism. The aetiology of hyperthyroidism/and thyrotoxicosis should be determined. Although the diagnosis is apparent based on the clinical presentation and initial biochemical evaluation, additional diagnostic testing is indicated. This testing should include: (1) measurement of thyroid-stimulating hormone receptor (TSHR) antibodies (TRAb); (2) analysis of thyroidal echogenicity and blood flow on ultrasonography; or (3) determination of radioactive iodine uptake (RAIU). A 123 I or 99mTc pertechnetate scan is recommended when the clinical presentation suggests toxic nodular goitre. A question arises regarding whether diagnostic workup and treatment (antithyroid drugs, radioiodine, surgery, and others) should be the same in children and adolescents as in adults, as well as whether there are the same goals of treatment in adolescents as in adults, in female patients vs in male patients, and in reproductive or in postreproductive age. In this aspect, different treatment modalities might be preferred to achieve euthyroidism and to avoid potential risks from the treatment. The vast majority of patients with thyroid disorders require life-long treatment; therefore, the collaboration of different specialists is warranted to achieve these goals and improve patients' quality of life.
\end{abstract}

\section{Key Words}

- hyperthyroidism

- thyrotoxicosis

- Graves' disease

- autoimmunity

- antithyroid drug

- surgery

- radioiodine

- thyroid ultrasound

- children

\section{Introduction}

Thyroid hormone level in normal ranges are necessary for appropriate neurocognitive development, growth, and metabolism at developmental ages. The following definitions related to thyroid hormones, thyroid hormones and their target tissues, such as hyperthyroidism, thyrotoxicosis, and Graves' disease, are given prior to further description of the management of these challenging thyroid states. Hyperthyroidism (in Latin: hyperthyreosis) is a medical condition in which excessive synthesis and secretion of thyroid hormones (triiodothyronine (T3) and/or thyroxine (T4)) into the blood are found. Thyrotoxicosis is a syndrome/set of clinical signs and symptoms caused by excessive action of thyroid hormones in tissues, the most common cause being their excessive tissue concentration. Graves' disease (GD) is a form of autoimmune hyperthyroidism associated 
with the presence of autoantibodies stimulating the thyroid-stimulating hormone (TSH) receptor (TRAb).

\section{Epidemiology}

GD accounts for $60-80 \%$ of all forms of hyperthyroidism in children and adolescents and according to some sources, might be responsible for $>95 \%$ of cases of hyperthyroidism; therefore, it can be considered almost synonymous with childhood and adolescent hyperthyroidism $(1,2)$. GD incidence varies between countries and may be rising $(3,4)$ with an annual incidence of 1 in 10,000 adolescents in the UK (5). Previous data referred to thyrotoxicosis rather than to GD. In a whole American paediatric population, the disease occurs with a prevalence of $1: 10,000$, compared to $1: 1000$ in adults (6). The onset of GD usually occurs during adolescence, and girls are 3-4 times more frequently affected than boys $(<4$ years of age, there is no sex dependence/predominance) (7). The second most frequent cause of hyperthyroidism is autoimmune thyroiditis (AIT), with its hyperthyroid phase termed hashitoxicosis. Both leading causes are of autoimmune origin in contrast to the remaining potential forms of hyperthyroidism of non-autoimmune origin, which are rare in the adolescent period but can occur in selected groups of patients.

\section{Aetiology and pathogenesis}

Hyperthyroidism occurs mainly in the course of autoimmune thyroid diseases (GD, including transient foetal/neonatal hyperthyroidism of maternal origin and the hyperthyroid phase of AIT). It is sometimes caused by less frequent non-autoimmune diseases of the thyroid gland (nodular toxic goitre, germline gainof-function mutation of the TSH receptor or Gs protein, pituitary adenoma secreting $\mathrm{TSH}$, amiodarone-induced hyperthyroidism, and receptor beta resistance to thyroid hormones).

GD is associated with the occurrence of TRAb stimulating the TSH receptor located in the thyroid cell membrane. The same mechanism of the disease is responsible for hyperthyroidism in newborns of mothers with active GD or following treatment but with persisting TRAb in the maternal blood passing through the placenta to the foetus. GD is dominated by thyroid gland hyperplasia and thyroid hormone hypersecretion. TRAb stimulates intact thyrocytes, and persistently increased levels of TRAb lead to hypersecretion of thyroid hormones as long as thyrocytes are capable of responding to this immune stimulus present within the thyroid. The occurrence of antithyroid peroxidase (TPOAb) and antithyroglobulin (TgAb) autoantibodies in patients with GD also indicates a coexisting cytotoxic process within the thyroid and the complexity of the pathogenesis of this form of hyperthyroidism. In other words, there is no 'pure/ isolated' GD in the patient, but it is always in a mixed form of autoimmune hyperthyroidism with the involvement of different mechanisms.

Hyperthyroidism can also occur in AIT (called hashitoxicosis since the goitrous form of AIT is termed as Hashimoto's disease), but its pathogenesis results from destruction of the gland with excessive release of prestored thyroid hormones.

That these two functionally opposite states, GD and AIT, can develop in the same person during different periods suggest that it is a dynamic process, very likely evolving under the influence of external factors operating in the local environment of the thyroid gland (8).

The underlying cause of autoimmune hyperthyroidism is genetic predisposition, as in other autoimmune diseases and triggering factors, such as infections, drugs, and environmental factors that are necessary to initiate the cascade of metabolic derangements from the silent to overt stage of a disease. Paediatric-onset GD and adult-onset GD share multiple common genetic risk variants. Kus and co-workers (9) demonstrated that HCP5 polymorphism is associated with an earlier age of GD onset in a dosedependent manner.

\section{Clinical picture}

The clinical picture of GD is dominated by a triad of symptoms described by Karl von Basedow as the so-called Merseburian triad: (1) goitre; (2) exophthalmos; and (3) tachycardia.

Initially, the course of GD in children can be scarcely symptomatic, with individual clinical symptoms increasing over many months. The clinical signs and symptoms of thyrotoxicosis are summarised in Table 1. Extrathyroidal manifestations of the disease, such as orbitopathy and dermopathy (pretibial myxoedema) are rarer in children than in adults and are less severe. These symptoms usually resolve with treatment and normalisation of the hormonal state. Prolonged exposure to excess thyroid hormones leads to an accelerated rate of growth and can also cause gonadotrophin-releasing hormone (GnRH)dependent precocious puberty, with a risk of potential 
Table 1 Clinical signs and symptoms of thyrotoxicosis.

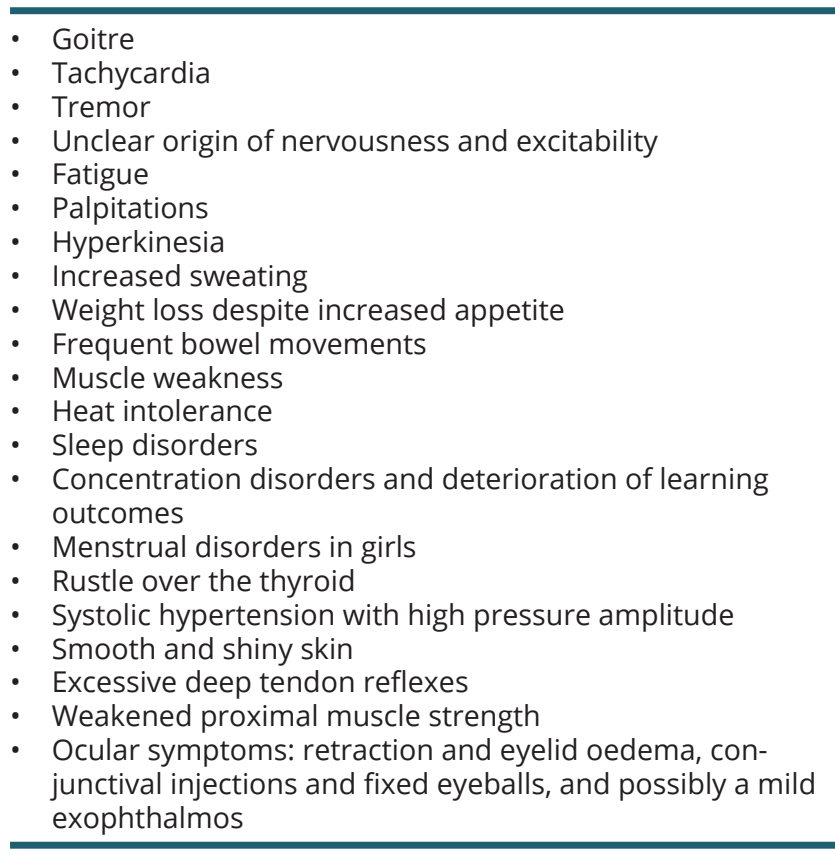

reduction in final height. In hashitoxicosis, the symptoms of overactivity usually persist for 2-8 weeks. In other forms of hyperthyroidism, the clinical manifestations and time duration are related to the advancement of the disease.

\section{Natural course}

Hyperthyroidism in GD progresses dynamically in the vast majority of patients if left untreated; however, spontaneous remission is also possible. Conversely, treatment of GD with antithyroid drugs (ATDs) does not prevent relapse of this disease.

Hashitoxicosis in its natural course switches to permanent hypothyroidism, usually over a few weeks/months. However, one should remember that hashitoxicosis can be recurrent.

The clinical course of other forms of hyperthyroidism is variable and difficult to predict, but there is certainly no spontaneous remission due to their organic nature.

\section{Diagnosis}

Apparent hyperthyroidism is characterised by reduced TSH concentrations and increased concentrations of the free thyroid hormones, fT4 and fT3. The cause of hyperthyroidism is supported by the assessment of serum TRAb, TPOAb, and TgAb. Blood pressure, heart rate, pulse rate, and electrocardiography are supplementary evaluations to be performed.

To assess the extent of hyperthyroidism and possible contraindications to pharmacotherapy, all patients should be examined for the following laboratory tests: total blood count with blood smear, serum lipid profile, glucose level, liver function tests (aminotransferases, alkaline phosphatase, bilirubin, and albumin), and urine analysis.

Thyroid ultrasound is of great importance in the assessment of patients with hyperthyroidism. In GD patients, it shows increased vascular flow on Doppler examination (colour Doppler (CD) and power Doppler (PD); Fig. 1) in addition to hypoechogenicity of the gland. Vascular flow can be a valuable tool in the subsequent follow-up of hyperthyroid patients.

To confirm and assess the severity of orbitopathy, an ophthalmological examination in each patient is recommended according to ETA/EUGOGO guidelines (10).

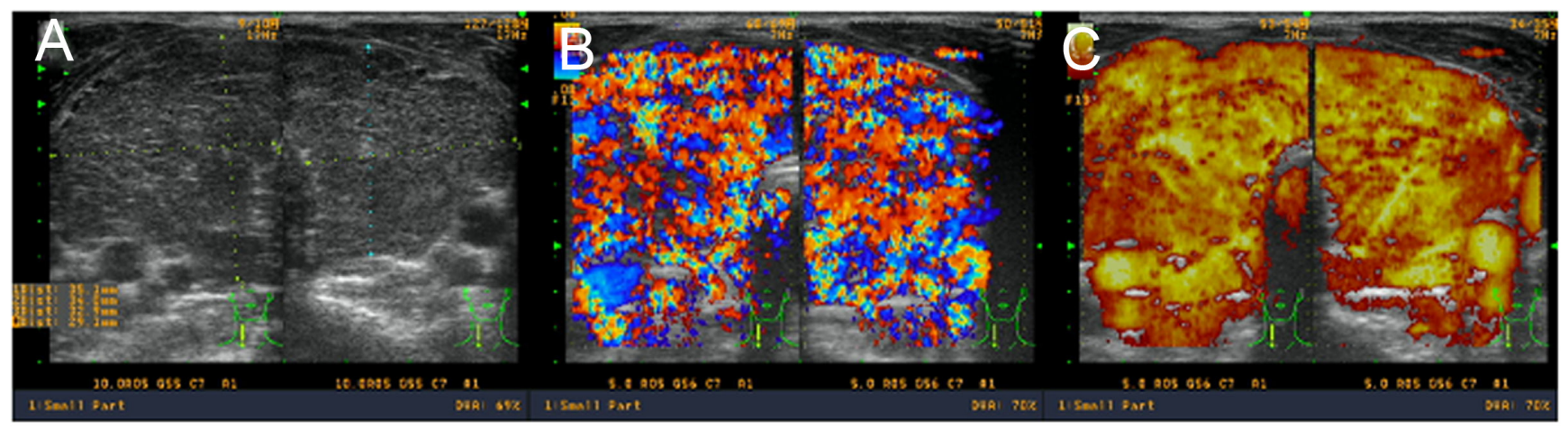

Figure 1

(A, B, and C) Thyroid ultrasound in a poorly controlled patient with Graves' disease. The gland is entirely enlarged, hypoechogenic (A), with a severely increased vascular flow in the colour Doppler (B), and power Doppler (C).

https://ec.bioscientifica.com https://doi.org/10.1530/EC-21-0191 (c) 2021 The authors Published by Bioscientifica Ltd

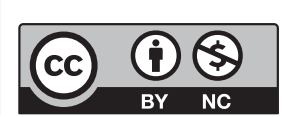

This work is licensed under a Creative Commons Attribution-NonCommercial 4.0 International License. 


\section{Differential diagnosis}

GD and hashitoxicosis should be differentiated from nonautoimmune causes of hyperthyroidism rarely found in children, such as the following:

1) Toxic nodular goitre (single toxic adenoma or multinodular toxic goitre) (11) is characterised (apart from single or multiple thyroid nodules) by reduced TSH concentrations; it can be accompanied by increased fT3 (T3-dependent thyrotoxicosis) and sometimes increased fT4 concentration; an ultrasound image that correlates with the clinical picture shows single or multiple foci, usually solid and with increased vascular flow on Doppler examination (CD/PD), in contrast to the parenchymal goitre in GD (although the coexistence of these two diseases is also possible); somatic gain-of-function mutations of TSHR, GNAS1 (12), or EZH1 (only in adults) (13) are found in these nodules.

2) McCune-Albright syndrome (MAS), a sporadic genetic disease, is caused by an activating mutation of GNAS1 for the Gs alpha membrane-associated protein, mediating the TSH-induced and other hormoneinduced activation of adenylyl cyclase; subclinical hyperthyroidism can be detected biochemically at birth or later in life.

3) Genetically determined hyperthyroidism associated with an activating (gain-of-function) mutation of the TSH receptor (congenital or late manifestation) is usually characterised by diffuse goitre and reduced TSH concentrations, which can also be accompanied by increased fT3 and sometimes increased fT4 concentrations (14); ultrasound usually shows a mildly reduced thyroid echogenicity; over time, multiple foci can appear, which are usually solid; vascular flow in the CD/PD can be mildly increased throughout the gland; the diagnosis is determined by a genetic test confirming the pathogenic germline variant of the TSH receptor gene (THSR) in patients with a negative TRAb results; and the genetic cause of hyperthyroidism due to TSHR gain-of-function mutations is rare and manifested in the foetal/neonatal period only in very severe states.

4) TSH-induced hyperthyroidism (pituitary adenoma secreting TSH; thyrotropinoma) is extremely rare in children and is usually characterised by diffuse goitre and increased serum concentrations of TSH, fT4, and fT3; and thyroid ultrasound can show mildly reduced echogenicity with mildly increased vascular flow on Doppler examination (CD/PD).
5) Amiodarone-induced hyperthyroidism is usually characterised by reduced TSH concentrations and increased fT3 and fT4 concentrations; and in some patients with TRAb presence prior to amiodarone treatment, classic GD can develop in coexistence with non-autoimmune thyroid overactivity due to high iodine intake $(15,16)$.

6) Iodine-induced hyperthyroidism (iodine-based syndrome) is a complication after iatrogenic overexposure to iodine-containing compounds, including drugs and contrast media used in imaging studies; it can develop in individuals with TRAb, as well as in patients with nodular, multinodular, or single autonomous nodules and is asymptomatic until increased iodine uptake by nodules/nodules occurs.

7) Resistance to thyroid hormones (RTH) syndrome (THRB only) is characterised by diffuse goitre with increased serum concentrations of fT4 and fT3 and inappropriately high normal, non-suppressed TSH levels (17); transient elevations of TSH might also be noticed since the diseases have quite sinusoid patterns; thyroid ultrasound shows mildly reduced echogenicity and mildly increased vascular flow in most patients on Doppler examination (CD/PD); these patients have attention deficit hyperactivity disorder, which is the first clinical manifestation in the majority of patients; and the genetic cause of hyperthyroidism due to THRB mutation is rare and manifests in the foetal/neonatal period only in a very severe state.

8) Iatrogenic hyperthyroidism is caused by an overdose of levothyroxine (LT4); goitre does not occur, but rather thyroid regression of treatment is prolonged; the serum shows increased fT4 and fT3 concentrations and reduced TSH levels; and it is not grouped with hyperthyroidism, since there is no overactivity of the thyroid gland.

The following procedures are also used in the differential diagnosis of non-autoimmune causes of hyperthyroidism: (1) thyroid ultrasound (US) (all forms of hyperactivity); (2) thyroid scintigraphy (toxic nodule/s); and (3) MRI of the pituitary/hypothalamic region (thyrotropinoma vs THRB defects) and genetic testing for TSHR, GNAS1, and THRB mutations ( $E Z H 1$ in adults), if available.

In a retrospective study of children from 22 centres in Turkey between 2007 and 2017, a total of 503 children were diagnosed with thyrotoxicosis. Of these children, $74.6 \%$ had been diagnosed with GD, 14.9\% with hashitoxicosis, and $10.5 \%$ with other less common causes of thyrotoxicosis (18).

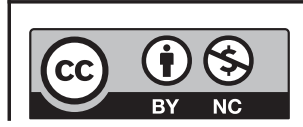

This work is licensed under a Creative Commons Attribution-NonCommercial 4.0 International License. 


\section{Treatment}

The following treatment modalities used in GD have remained largely unchanged for the past 80 years:

1) Pharmacotherapy with ATDs as monotherapy (dose titration (DT) in a smaller dose tailored to render the patient euthyroid or in combination with LT4 (block and replace (BR)) with a blocking dose of ATD and LT4 replacement.

2) Two definitive options - (a) surgery and (b) radioiodine (I-131 (RAI)) - followed by life-long treatment with LT4.

The treatment of other, less frequent forms of hyperthyroidism in children depends on their cause and whether coexisting thyroid nodules are present.

\section{Pharmacotherapy}

The primary treatment of hyperthyroidism includes the use of ATDs from the thionamide group, and the first choice/line drugs are imidazole derivatives (usually thiamazole (MMI)). With the use of an average/middle dose $(\sim 0.5 \mathrm{mg} / \mathrm{kg}$ bw/day of MMI in two to three doses per day), undesirable effects are rare. Propylthiouracil (PTU), very often used in the past in the United States, has not been used in the treatment of children since 2009 due to the risk of induction of acute liver failure and even death (19). The only exception in which PTU is possibly allowed to treat children for a short period of time, is an allergy to MMI or other drug intolerance, making it impossible to prepare the patient for radical treatment. The major advantage of ATD treatment is that normal homeostasis of the hypothalamus-pituitary-thyroid axis can be restored, with periods of drug treatment followed by freedom from medical intervention. An area of controversy also involves the optimal duration of ATD therapy. For some children, the prolonged use of ATDs is a valid approach (20), but for most, this approach will not increase the chance of remission (21). Historically, most children with GD treated with ATDs do not experience remission, but most remissions do not end in relapse. Adverse reactions to MMI are common, but generally mild (22). Adverse events of ATDs (Table 2) could likely be less frequent if we did not use a high dose of MMI ( $\geq 30 \mathrm{mg} /$ day) and if based on pharmacokinetics, the dose of MMI is divided into two to three doses daily. A randomised trial in 81 young patients aged 2-17 years old with newly diagnosed thyrotoxicosis at 15 UK centres showed no evidence suggesting that BR is associated with improved biochemical stability compared
Table 2 Adverse effects of antithyroid drugs.

Adverse effects that require the immediate cessation of thionamide treatment:

1) Agranulocytosis ( $<500 / \mu \mathrm{L} ; 0.2-0.5 \%$ treated), aplastic anemia (even rarer)

2) Acute hepatitis (sporadic; mainly after PTU), cholestatic jaundice (after thiamazole)

3) Vasculitis with antibodies to neutrophil cytoplasm (ANCA) and lupus-like syndrome.

4) Acute pancreatitis

Adverse effects that do not require the immediate cessation of thionamide treatment (a dose reduction or change of medication may be sufficient):

1) Pruritus, urticaria (1-5\%), sometimes very severe skin symptoms - they can be controlled with antihistamines or by changing the antithyroid drug

2) Muscle and joint pain (in arthritis, found in 1-5\% of cases, most often it is necessary to stop treatment)

3) Taste disorders

4) Nausea and vomiting (depending on the dose, it is worth using the drug in divided doses)

5) Increase in serum aminotransferase activity (up to $30 \%$ of patients treated in the past with PTU; a mild increase does not necessarily lead to treatment discontinuation)

6) Transient granulocytopaenia (1-5\%) or thrombocytopaenia $(<1 \%)$ may disappear after the drug dose has been reduced:

a) $1500-1000 / \mu \mathrm{L}$ - more frequent controls are recommended, consider a dose reduction of ATD

b) $1000-500 / \mu \mathrm{L}$ - reduce the dose or consider stopping the drug.

to DT (23). In another study (24), 28 paediatric GD patients with unstable responses to ATDs were treated with LT4 and a medium dose of ATD. The patients showed better control of thyroid function during BR therapy, presenting fewer episodes of hyperthyroidism and hypothyroidism. No serious side effects during the BR approach were observed. The BR method did not increase the remission rates and could be used when GD is difficult to manage with methimazole alone. It might represent a therapeutic option to postpone definitive treatments until a suitable age. The patients in this study received 25-50 $\mu$ g of LT4, which was a supplementary, and not a replacement dose for their age. In recent studies $(25,26)$, approximately $40-50 \%$ of 154 newly diagnosed children with GD achieved remission after prolonged treatment with ATDs for several years (8, $9,10)$. Long-term ATD treatment of 300 patients remained an effective treatment option for GD in children. Paediatric GD patients were more likely to achieve remission if they met the following criteria: (1) aged <5 years old; (2) having no family history of thyroid disease; and (3) having initial lower TRAb levels (27). Severe hyperthyroidism, with particularly high TRAb concentrations at diagnosis, could facilitate the identification of patients requiring regular serum fT3 determinations and potentially requiring

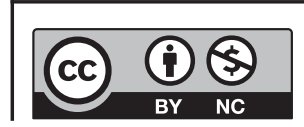

This work is licensed under a Creative Commons Attribution-NonCommercial 4.0 International License. 
higher ATD dosages during follow-up (28). A recent systematic review showed that the overall remission rate in methimazole-treated paediatric GD is $28.8 \%$. A few small studies indicate that longer treatment increases the remission rate. However, evidence is limited and further research is necessary to investigate the efficacy of longer treatment durations (29).

In the author's opinion, based on 25 years of personal experience, the commonly used and preferred monotherapy with ATDs ('titration' method) is not an optimal treatment for hyperthyroidism at developmental ages. Conversely, the literature data do not support that combined therapy (ATD plus LT4) is superior to monotherapy. The increases in length/height and body weight, as well as the child's maturation process (puberty), make it necessary to periodically adapt the dose of the ATD to the dynamically developing organism, resulting in difficulties in achieving normalization of TSH, fT4, and fT3. Many patients treated in this manner (DT) often experience prolonged TSH suppression, very likely due to insufficient control of excessive thyroid hormone biosynthesis and release. In contrast, a significantly reduced fT4 concentration and a compensatory increase in fT3 concentration falsely suggest euthyroidism and might not allow for recovery of normal functioning of the thyroid axis.

Combined therapy with and the use of remissionsupporting doses of ATD and LT4 to achieve optimal levels of free thyroid hormones and appropriate TSH levels allow these patients to control the underlying disease more effectively, and they treat hypothyroidism as a result of ATD treatment. They also allow us to avoid complications in the organs of vision thanks to systematic regression of TRAb, followed by regression of thyroid eye disease, and they additionally allow for better weight control (30) (M Niedziela, unpublished observations). Treatment with ATDs should be administered very carefully, avoiding long-term intervals to avoid hypothyroidism and the resulting risks. The frequency of control visits and the proper adjustment of both agents (ATD and LT4) are of great importance to reaching a successful balance. The recovery of the pituitary-thyroid axis is extremely important (crucial in my opinion) for effective therapy and monitoring. If the patient has a suppressed $\mathrm{TSH}$ concentration during treatment, it indicates that the underlying disease has not been controlled. In some patients, TSH suppression is persistent although fT4 levels are low normal or less than the normal range, which might be a result of long-term exposure to high concentrations of thyroid hormones prior to initial treatment; therefore, one should be patient in expecting full recovery of the axis. Conversely, a sudden increase in TSH concentrations due to profound hypothyroidism (when the pituitarythyroid axis is unblocked) must be prevented to avoid causing a significant development of goitre and not increasing the risk of orbitopathy. Hypothyroidism, due to the accumulation of water by glycosaminoglycans in the retroorbital tissues, can also worsen thyroid eye disease.

I no longer use the term 'BR' but 'control and supplement' because, with the proposed ATD treatment, we do not completely block thyroid hormone synthesis but efficiently inhibit and control thyroid function, and we do not substitute with the replacement dose of L-thyroxine but supplement with a lower-than-replacement L-thyroxine dose, according to the controlling ATD dose and based on fT4, fT3, and TSH levels.

In the initial stage of hyperthyroidism therapy, $\beta$-blockers at a dose of $1 \mathrm{mg} / \mathrm{kg}$ bw/day in two to three doses are administered to control tachycardia and arrhythmia, hand trembling, increased sweating, and to reduce eyelid retraction.

\section{Definitive treatment (surgery vs radioiodine)}

The aim of definitive treatment is to achieve permanent hypothyroidism. This goal can be achieved by excision of the entire thyroid or destruction of the gland with RAI (I-131). According to most guidelines and expert opinion, the preferred treatment for hyperthyroidism in childhood and during the period of adolescence is total thyroidectomy (TT), and after adolescence, RAI treatment can be considered (20). In American patients operated on with GD, approximately $60 \%$ of diagnosed thyroid carcinomas were found incidentally on postoperative histological examination of the thyroid gland, both in children (31) and in adults (32).

Therefore, choosing a TT throughout the developmental period is safer because there is a risk of accidental detection of thyroid microcarcinoma. In addition, adolescent girls and young adult women during the child-bearing period are very likely to have high TRAb levels in their blood after RAI administration, with further risk of foetal and neonatal thyrotoxicosis.

Surgical treatment (TT) is the preferred definitive method with the following indications: (1) children $<10$ years old or rather throughout the entire developmental age; (2) glands of significant size/volume (>50-80 g); (3) severe/moderate orbitopathy; (4) lack of iodine uptake; and (5) coexistence of a tumour-like lesion suspected of neoplasm/cancer. 
In a study of 58 children with GD and 108 children with hereditary C-cell disease who underwent TT, children with GD had a 1.7-fold greater overall risk of transient hypoparathyroidism than children with hereditary C-cell disease. Transient palsy of recurrent laryngeal nerve, wound haemorrhage, and wound infections infrequently resolved spontaneously and after reoperation. In summary, the risk of permanent side effects was minimal in the experienced hands of high-format surgeons (33). The analysis of 464 children, including 124 GD patients who underwent thyroidectomy at a single centre, showed low complication rates, emphasizing that referral to highvolume centres should be considered for children and adolescents with thyroid disease requiring surgery (34). In another study of paediatric thyroidectomy for GD, the authors found that indications for surgery were similar for children and adults, except that no children who underwent surgery had previously failed RAI therapy. They did not find that children were treated for longer periods of time with medications than adults and reported that permanent complication rates of surgery were low in both children and adults when performed by high-volume surgeons (35). Based on the current literature data from surgery departments, we should not be concerned about the safety of TT (36). The novel option for children is outpatient thyroid surgery with discharge home within 6 $\mathrm{h}$ after completion of the operation. It is safe and effective for paediatric patients, as it is in adults (37).

Successful treatment with TT can be evaluated by neck ultrasound exploring for thyroid tissue remnants and by thyroglobulin measurement. The latter could serve as an optimal diagnostic tool to demonstrate the lack of thyroid tissue. Subsequent measurement of TRAb is a wonderful predictive tool for potential thyroid eye disease regression. In experienced hands of the high-volume surgeon, TT is safe and effective, leading to permanent hypothyroidism, no further cancer risk, a significant decline in or normalization of TRAb levels, regression of prior thyroid eye disease and no maternal TRAb-related consequences for the foetus and neonate.

In other patients, RAI in ablative doses could be proposed. In female patients of child-bearing age, a pregnancy test should be performed prior to the planned RAI treatment because pregnancy is a contraindication to RAI treatment (also breastfeeding). The indication for RAI can be particularly considered:

1) If granulocytopenia or thrombocytopenia after ATD treatment is present.
2) Disorders of the haematopoietic system occurring independently of GD are diagnosed before treatment with ATD.

A 36-year retrospective analysis of the efficacy and safety of RAI in treating 116 young GD patients between 1953 and 1973 showed that none of the patients developed cancer of the thyroid or leukaemia. Early in the study, when the objective of treatment was euthyroidism, the dose of radioiodine was low, relapses were frequent, and retreatment was needed. Pregnancies did not result in an unusual number of congenital anomalies or spontaneous abortions (38). Because of the increased risk of thyroid cancer associated with low-dose thyroid irradiation in children, larger, rather than smaller, doses of I-131 should be administered. Potential long-term adverse side effects, including thyroid cancer and genetic damage, have yet to be observed in individuals treated as children or adolescents with I-131 (39). On evaluation of clinical progression after RAI therapy, Cury et al. (40) reviewed 65 medical records of GD patients $<19$ years old. They found that $80 \%$ of patients developed hypothyroidism $(30.0 \%$ within 3 months after RAI, 48.0\% within 6 months, 5.7\% $>6$ and $<12$ months, and $15.3 \%$ patients $>12$ months). Eight of $65(12.3 \%)$ patients had euthyroidism 6 months after RAI therapy, and 5 of 65 (7.9\%) patients did not achieve remission 7 months after RAI therapy. In eight patients, a second RAI dose was necessary. Another retrospective review of GD patients who underwent 78 RAIs showed that 73\% became hypothyroid, 4\% euthyroid and 23\% overt or subclinical hyperthyroid. Patients with smaller thyroid sizes and an I-131 dose close to $250 \mu \mathrm{Ci} / \mathrm{g}$ of thyroid tissue have a greater likelihood of achieving hypothyroidism. Most patients remained hyperthyroid 1 month postRAI, but by 3 months, the majority became hypothyroid. Testing at 2-3 months after RAI is most helpful for confirming the response to RAI (41). In a retrospective and multifactorial data analysis of 32 children and adolescents with hyperthyroidism who had prior treatment with ATDs, the authors concluded that RAI therapy with an I-131 dose of $250-400 \mu \mathrm{Ci} / \mathrm{g}$ of thyroid tissue might be suitable in patients with medical failure from ATDs (42). Radioiodine ablation has been proposed as the main alternative therapy, although surgery could have some advantages if performed in a high-volume centre; using higher weight-based doses of I-131 $(250 \mu \mathrm{Ci} / \mathrm{g}$ thyroid tissue) could increase the proportion of patients achieving hypothyroidism and decrease repeat ablations (43). Ballal et al. (44), in a retrospective study of 114 children with 
GD, concluded that prior ATD (carbimazole) treatment did not alter the outcomes of radioiodine therapy in paediatric GD.

Given the published benefits and perceived low risks of RAI when compared to surgery or long-term ATD treatment, the trend towards therapy with RAI is likely to continue.

An example of inappropriate use of I-131 is the case of an 11-year-old girl with newly diagnosed GD who received RAI for definitive treatment of her hyperthyroidism. Within 24 h of receiving I-131, the patient developed a lifethreatening thyroid storm with long-term neurological complications (45). Therefore, I strongly state that such an approach with RAI treatment in newly diagnosed paediatric GD should never be considered. Each patient with clinical and hormonal hyperthyroidism should receive pretreatment with ATDs to eliminate the potential risk of postablative thyroid storm.

The initial surge of TRAb soon after an I-131 ablative dose followed by their unpredictable timing of decline might incur risk of TED exacerbation in GD patients. The majority of patients with GD gradually enter remission of TSH-receptor autoimmunity during medical treatment or after surgical therapy, with no difference between the types of therapy. Remission of TSH-receptor autoimmunity after radioiodine therapy is less common (30).

Based on this postRAI surge of TRAb, a high preRAI concentration of TRAb is a relative contraindication to RAI treatment due to the risk of occurrence or aggravation thyroid orbitopathy (46).

The long-term outcomes of RAI therapy (median, 13.0 $\mathrm{mCi}$ ) for 117 juvenile Japanese GD patients were analysed in a retrospective study. I-131 administration was performed once in 89 patients, twice in 26 patients, and three times in 2 patients. At the latest follow-up more than 12 months after RAI $(n=111)$, the patients' thyroid functions were overt hypothyroidism (91\%), subclinical hypothyroidism (2\%), normal (5\%), or subclinical hyperthyroidism (2\%). New thyroid nodules were detected in nine patients 4-17 years after initial RAI. Patients with newly detected thyroid nodules underwent RAI with lower doses of I-131 and had larger residual thyroid volumes than those without nodules. None of the patients were diagnosed with thyroid cancer or other malignancies over a median follow-up period of 95 months. RAI was found to be effective and safe in juvenile GD. However, cumulative evidence from further studies is required to confirm the long-term safety of RAI for juvenile GD (47). Medical records from 81 children and adolescents with GD showed that RAI is a safe and effective mode of treatment with a relapse rate of $12.1 \%$, in contrast to effective TT (0.0\% relapse), but with $20 \%$ permanent hypoparathyroidism. Therefore, it is not surprising that RAI is recommended as the first-line treatment in such centres if a high-volume surgeon is not available (48).

Whether radioiodine is ideal for adolescents with a subsequent rise in TRAb and potential foetal/neonatal complications should be addressed before this treatment modality is discussed. The potential clinical outcomes related to RAI are shown in Fig. 2. Therefore, in Fig. 3, I would like to propose a therapeutic flowchart for hyperthyroidism in the course of GD in children/adolescents in a tertiary centre.

\section{Treatment of other forms of hyperthyroidism}

In the case of toxic nodular goitre, initial ATD treatment is usually necessary to attain euthyroidism followed by surgery, depending on the tumour/s location and

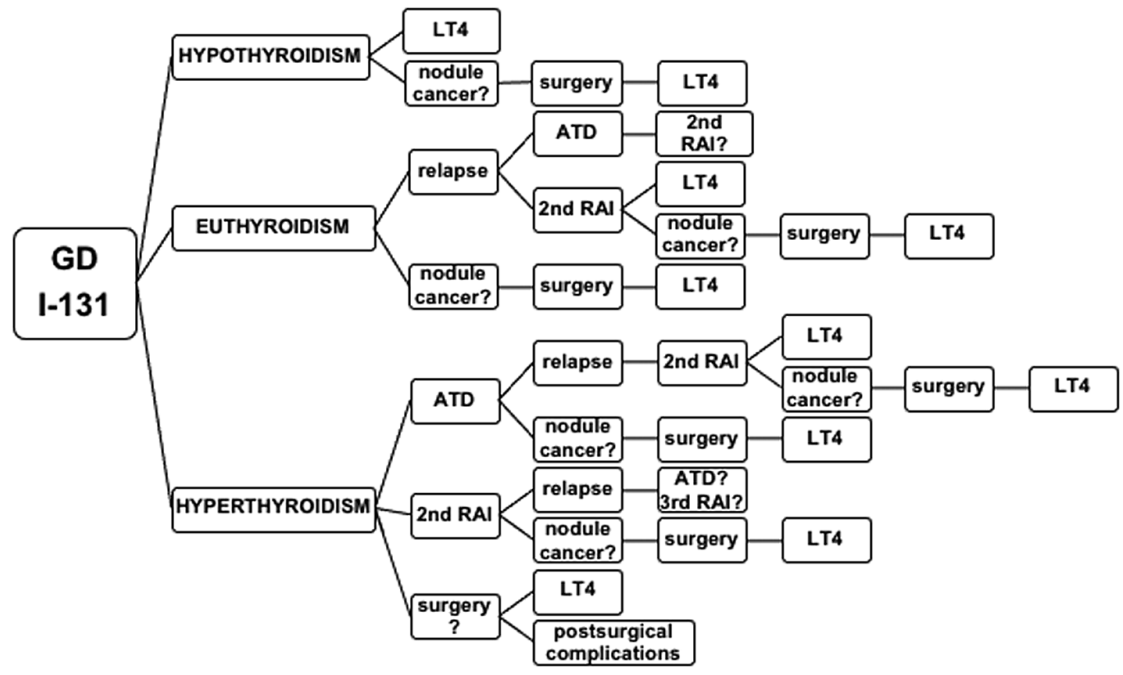

Figure 2

RAl treatment in Graves' disease and possible outcomes. (c) 2021 The authors Published by Bioscientifica Ltd

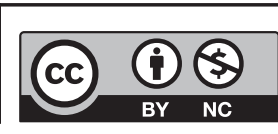

This work is licensed under a Creative Commons Attribution-NonCommercial 4.0 International License. 


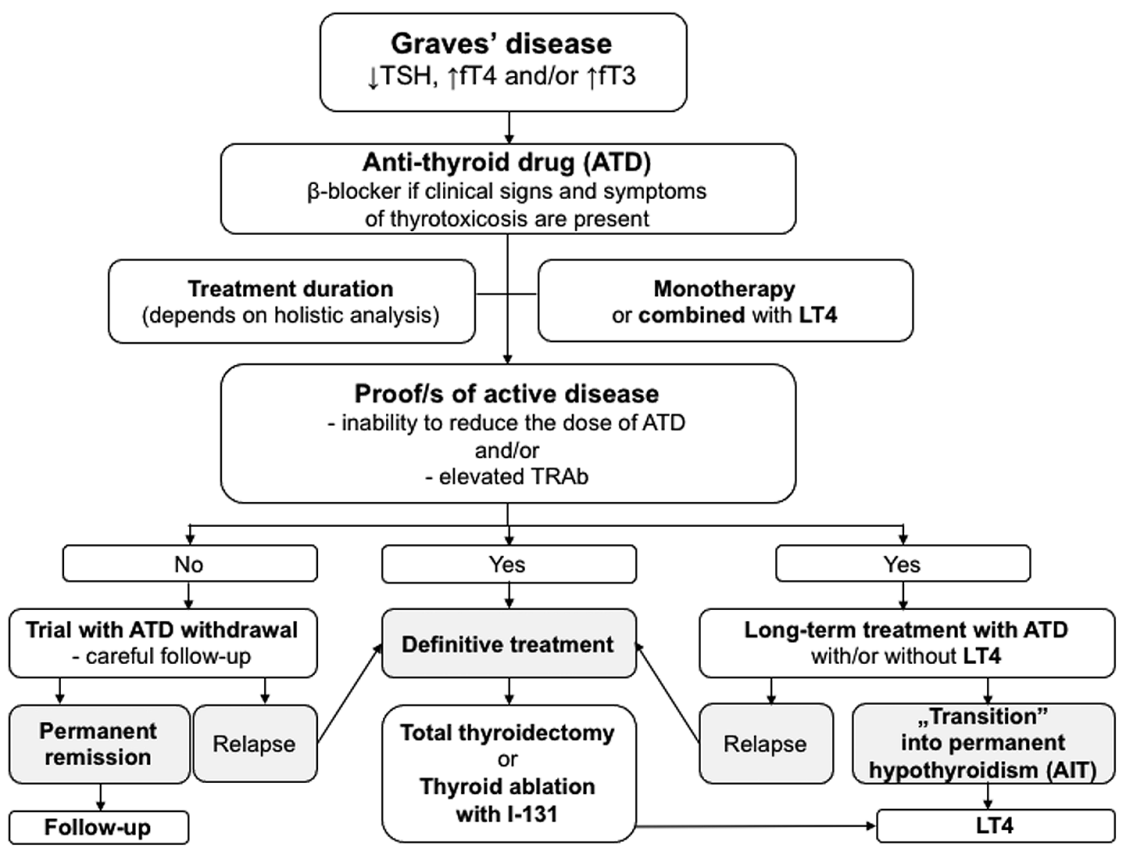

Figure 3

The therapeutic flowchart for Graves' disease hyperthyroidism in children/adolescents. lobectomy vs TT. In the case of thyrotropinoma, initial treatment with somatostatin analogues is the treatment of choice, followed by neurosurgery and total resection of the tumour. Treatment of amiodarone-induced hyperthyroidism depends on the type: ATD in type I or glucocorticosteroids in type II (15). Patients and clinicians should be aware of the impact of amiodarone on thyroid function during and in the months and perhaps years after treatment (16). Thyroid status should be evaluated prior to amiodarone treatment and thereafter at control visits.

Clinical and biochemical pictures of hyperthyroidism in children can significantly change according to its aetiologies. GD and AIT are responsible for 84 and 12\%, respectively, of all cases of hyperthyroidism in childhood. Hyperfunctioning thyroid nodules (3\% of cases), TSHsecreting adenomas ( 1\%), and McCune-Albright syndrome $(\sim 1 \%)$ are distinctly less common. Therefore, (1) hyperthyroid syndrome in childhood can present with very different clinical pictures, which are specifically related to the respective aetiologies; and (2) diagnostic procedures and therapeutic management are not the same in the various conditions (49).

\section{Follow-up}

In the author's opinion, the proposed minimum period of conservative treatment of GD for $18-24$ months $(1,50)$ or 36 months (16) is not sufficient to decide whether to stop
ATD treatment or not (Fig. 3). The decision of whether to stop treatment with ATDs should be made based on the clinical picture; TSH, fT4, fT3, and TRAb levels; and thyroid ultrasound with special awareness of its echogenicity and blood flow. The discontinuation of ATD therapy can be considered if the result of two consecutive measurements of TRAb, performed in an interval of 6 months, is within the reference value (negative result). Elevated TRAb levels indicate that the risk of relapse within a few weeks after stopping ATD treatment is high. The guarantee of good control of a patient with hyperthyroidism is the frequent determination of free thyroid hormones and TSH levels in serum in the initial phase of treatment so that, after hormonal normalization (return to normal functioning of the pituitary-thyroid axis), control visits can be planned every 3-4 months or even every 6 months. Such a visit plan significantly improves the quality of life of patients. Treatment of each patient should be highly individualised, not proposing fixed intervals, because patients have different initial states and therefore require adequate therapeutic and controlling approaches.

Choosing a treatment approach for childhood GD is often a difficult and highly personal decision. Discussion of the advantages and risks of each therapeutic option is essential in helping the patient and family to select a treatment option $(50,51)$. De Luca and Valenzise (52) also recommend individualising the therapeutic approach without prejudice towards radical therapies that become necessary in cases of relapse or adverse effects or poor
This work is licensed under a Creative Commons Attribution-NonCommercial 4.0 International License. 
compliance with ATDs. The optimal approach also depends on patient or family preferences and specific patient clinical features (50).

When I-131 is administered, dosages should be greater than $150 \mathrm{uCi} / \mathrm{g}$ of thyroid tissue, with higher dosages required for large glands. Considering that there will be low-level whole-body radiation exposure associated with I-131, this treatment should be avoided in young children. When surgery is performed, TT is the recommended procedure. Complications of thyroidectomy in children are considerably more common than in adults; thus, an experienced thyroid surgeon is needed when children are operated on (6).

Near-total thyroidectomy is not a perfect decision since thyroid tissue remnants can still be immunogenic in TRAb production, maintaining the risk for relapse and TED and nodule formation.

The criteria for full remission in GD should be same as the characteristics of healthy subjects: (1) no goitre, with clinical euthyroidism; (2) serum concentrations of TSH, fT4, and fT3 within normal ranges; (3) negative results of TRAb; and (4) normoechogenic thyroid gland on ultrasound without increased blood flow. These criteria are not fulfilled if GD patients switch to autoimmune thyroiditis.

The ATA defined remission as being euthyroid for 1 year after cessation of therapy (1). Treatment with ATDs can be continued over long periods of time; however, it is important to remember that the rare but severe side effects of ATDs are dose dependent and that early detection requires periodic monitoring.

\section{Special states}

\section{Graves' disease and the risk of thyroid cancer}

In more than 250 children $<19$ years old diagnosed with a solitary nodule or multinodular goitre in 1996-2000, the authors did not detect a single case of thyroid carcinoma in patients with an autoimmune disorder (GD or AIT) (53). A single case of thyroid carcinoma was found in a 14-year-old boy with GD who did not adhere to longterm treatment with ATDs but was found at the time of diagnosis of GD (54). Further literature data also showed a single but rather aggressive papillary thyroid carcinoma with metastases to the lungs in a 12-year-old girl with GD treated for 4 years with ATDs (55). The tumour was not manifested at disease onset. A similar case report was described by Kojima-Ishii et al. (56) in a 14-year-old girl who developed minimally invasive follicular thyroid carcinoma treated with ATDs for 4 years. Concurrent differentiated thyroid carcinoma (DTC) occurs in paediatric GD patients. DTC was identified in $22 \%$ of the GD patients. Preoperative fine needle aspiration biopsy detected four concurrent DTCs, preoperatively. Thyroid ultrasound was found to be an efficient tool for selecting patients for thyroidectomy vs RAI ablation (57). In contrast, MacFarland et al. (31) found that concurrent DTC in paediatric patients with GD is not associated with a greater disease burden at presentation and shows no significant differences in 2-year outcomes compared with DTC alone. A retrospective analysis was performed of 233 GD patients who underwent thyroidectomy, and pathology reports were reviewed for coexistent nodules. A total of 103 had one or more thyroid nodules, which were incidentally discovered on pathologic exam in 54 patients. Fourteen patients had cancer, nine had papillary microcarcinoma, and five had papillary cancer $\geq 1 \mathrm{~cm}$ that occurred within a nodule diagnosed preoperatively. Most thyroid nodules in GD patients were incidentally discovered on pathologic exam. A higher rate of malignancy was found in patients with GD and clinically identifiable nodular disease (32). Therefore, it is not surprising that the association between GD and thyroid carcinoma remains controversial. In the study by Jia et al. (58), the authors aimed to investigate incidental thyroid carcinoma (ITC) in surgery-treated hyperthyroid patients with and without GD. Their study demonstrated that GD was not associated with an increased risk of ITC in surgery-treated hyperthyroid patients.

The question therefore arises regarding whether GD was the switching-on mechanism and thyroid cancer was a secondary result or whether there was some other pathogenetic link between the two disorders $(54,59,60)$. Finally, were the two disorders of independent origins? Is the RAI dose used in children with GD sufficiently powerful to kill cancer cells, such as those in incidentally found microcarcinomas?

\section{Subclinical hyperthyroidism (hypothyrotropinaemia)}

Sometimes, the so-called subclinical hyperthyroidism is observed, in which only suppressed serum TSH is found. Concentrations of thyroid hormones remain normal, no goitre is observed, thyroid ultrasound is normal, and the patient remains euthyroid. This condition does not necessarily indicate thyroid pathology and can occur in healthy individuals. However, analogous to subclinical hypothyroidism, so-called poorly manifested, rather than

This work is licensed under a Creative Commons Attribution-NonCommercial 4.0 International License. 
asymptomatic, hyperthyroidism should be considered. It should be assessed whether subclinical hyperthyroidism is not a scantily symptomatic hyperthyroidism leading to concentration disturbances, sleep disturbances, poorer learning outcomes, progression of goitre, accelerated puberty, and acceleration of bone age, with the risk of deterioration of the predicted final growth. Therefore, for safety reasons, a short-term clinical and laboratory follow-up (3-6 months) seems to be necessary to avoid missing a defined cause.

\section{Thyroid eye disease (thyroid orbitopathy)}

Thyroid orbitopathy (Graves' orbitopathy) or thyroid eye disease (TED) is a set of ocular symptoms caused by immunological inflammation of the soft tissue of the orbit in GD, leading to temporary or permanent damage to the organs of vision. This condition is mainly related to excess thyroid hormones and their effects on the sympathetic nervous system and is usually mild. The symptoms of TED are less pronounced in children than in adults and are systematically regressive with properly administered primary treatment (ATDs) or secondary (TT). Orbitopathy in children and adolescents rarely requires additional treatment. TED usually has mild manifestations in pre- and postpubertal paediatric patients, and orbital decompression is rarely necessary. The question arises how prior management can prevent this advanced and severe TED (61).

\section{Thyroid storm}

A thyroid storm is a state of sudden and violent collapse of body homeostasis. It develops on the basis of undiagnosed or insufficiently/inappropriately treated hyperthyroidism. It co-occurs with disturbances of consciousness up to and including coma, heart failure and shock symptoms, and high body temperature. Thyroid storms are extremely rare in children and adolescents.

\section{Hyperthyroidism in neonatal period}

The appearance of hyperthyroidism in the newborn due to the passage of TRAb from the mother with GD depends on whether the pregnant woman used ATDs. The risk of overt hyperthyroidism exists as long as TRAbs remain in the circulation of the newborn or infant (usually after 3 months of age, they are no longer found). If the pregnant woman received ATDs and her disease was well controlled, hyperthyroidism would manifest itself in the newborn a few days after birth, when ATDs taken by the mother (which effectively block foetal thyroid hormone production) declined in the newborn's blood and stopped working. In contrast, a newborn of a mother with TRAb present in her blood, untreated or treated before pregnancy, will develop apparent hyperthyroidism immediately after birth (it can occur during foetal life). Such a neonate requires immediate therapy with an ATD and $\beta$-blocker on the 1st day of postnatal life and but rarely in foetal life.

Due to the increasing incidence of autoimmune thyroid diseases in adolescent and older female patients, it is necessary to properly monitor the hormonal status and autoimmune profiles before, during, and after pregnancy to achieve the optimal state and development of the foetus, newborn, and infant.

A TSH receptor defect caused by an activating mutation sometimes appears clinically in the neonatal period.

\section{Management during the COVID-19 pandemic}

Autoimmune thyroid diseases are not linked to increased risk of COVID-19, but uncontrolled thyrotoxicosis can result in more severe complications from SARS-CoV-2 infection, including thyroid storms. The authors of guidelines have proposed that the management of patients with a new diagnosis of hyperthyroidism is best undertaken with a BR regimen due to limited biochemical testing and ultrasound availability. ATD-induced neutropenia might favour the progression of COVID-19, and symptoms of infection could be confused with SARS-CoV-2 infection; therefore, in cases of flu-like manifestations, measurement of neutrophils should be considered (62).

One year after the onset of the COVID-19 pandemic and when physicians were vaccinated, we should bear in mind that frequent biochemical testing is crucial for proper treatment of GD hyperthyroidism. The roles of thyroid ultrasonography and biopsy cannot be limited, and the determination of malignancy risks associated with palpable thyroid nodules in children cannot be postponed.

\section{Prognosis}

The following risk factors for the relapse of GD were proposed $(63,64,65)$ : (1) onset of disease <12 years old; (2) a large initial thyroid volume $(>2.5 \times$ normal size for age and sex); (3) high initial concentration of TRAb; (4) high initial concentration of fT4 $>4 \mathrm{ng} / \mathrm{dL}$ (50 pmol/L); and (5) non-white race.

This work is licensed under a Creative Commons Attribution-NonCommercial 4.0 International License. 
Since a very small percentage of permanent remissions is observed after ATD treatment, this treatment should be considered, rather than a form of preparation for a definitive treatment. Long-term ATD treatment with/ or without LT4 can be an effective and safe treatment for GD. It is also possible to 'switch' from GD to autoimmune thyroiditis. Both diseases, GD and AIT, can switch from one to another, so it seems that these two diseases are in fact two faces of potentially the same disorder - a mixed autoimmune thyroid disease that involves lymphocytic thyroid infiltration of the gland and serum autoantibodies. Children and adolescents can therefore be offered an attempt at long-term treatment with ATDs because of the possibility of 'transition' to autoimmune inflammation with subsequent hypothyroidism and further treatment with LT4 only, thus avoiding definitive treatment (Fig. 3). Early initiation of radical treatment does not provide an opportunity to observe such a course of autoimmune hyperthyroidism. Full remission in the patient does not guarantee its maintenance if strong exposure to stress occurs. However, these patients remain at risk for thyroid nodules, including cancer, like other patients with AIT, and might still require TT in the future. Therefore, longterm follow-up with ultrasound is mandatory since the increased consistency of the thyroid in the majority of patients does not allow for the detection of palpable lesions, even in experienced hands $(59,60)$.

In summary, we now have available four management options: ATDs, surgery, radioiodine, and switch to permanent hypothyroidism, followed by L-thyroxine therapy.

\section{Impact on life activity}

Hyperthyroidism adversely affects the patient's performance at school but is not a contraindication for attending school after controlling the acute phase of the disease. Due to impaired concentration, sleep disturbances and abnormal metabolism, it is extremely important to reduce the demands on the patient until euthyroidism is reached. Until then, due to accompanying fatigue inadequate for physical effort, these patients should be exempted from physical education classes. However, there are no contraindications regarding professional choices in patients with properly controlled disease.

\section{Novel future strategies and conclusions}

New therapeutic options for the treatment of GD and TED are expected. The current novel therapies under investigation include biologics (to influence B lymphocyte immunomodulation), small molecules (to block immunoglobulin recycling or regulate TSH receptor signalling by blocking TRAb or using TSHR antagonists), and peptides immunomodulating TSHR (regulating immune tolerance). In particular, TSHR-targeted treatment modalities seem to be of the greatest importance since the current protocols remain insufficient for attaining permanent remission and limited adverse events (66).

The superior goal is to treat less aggressively, with less toxicity, and with a better final outcome. I hope that novel therapeutic proposals have the potential to be incorporated into clinical practice in the near future and significantly improve the quality of life of our patients.

\section{Declaration of interest}

The author declares that there is no conflict of interest that could be perceived as prejudicing the impartiality of this review.

\section{Funding}

This review did not receive any specific grant from any funding agency in the public, commercial or not-for-profit sector.

\section{Acknowledgements}

Niedziela M 2020 Hyperthyroidism across the life span: hyperthyroidism in adolescents. Presented at 22nd European Congress of Endocrinology ESE, online 05 September 2020 to 09 September 2020. Endocrine Abstracts 2020 70 S5.3. (https://doi.org/10.1530/endoabs.70.S5.3).

\section{References}

1 Ross DS, Burch HB, Cooper DS, Greenlee MC, Laurberg P, Maia AL, Rivkees SA, Samuels M, Sosa JA, Stan MN, et al. American Thyroid Association guidelines for diagnosis and management of hyperthyroidism and other causes of thyrotoxicosis. Thyroid 2016 26 1343-1421. (https://doi.org/10.1089/thy.2016.0229). Erratum in: Thyroid 201727 1462. (https://doi.org/10.1089/thy.2016.0229.correx)

2 Cheetham T \& Bliss R. Treatment options in the young patient with Graves' disease. Clinical Endocrinology 201685 161-164. (https://doi. org/10.1111/cen.12871)

3 Wong GW \& Cheng PS. Increasing incidence of childhood Graves' disease in Hong Kong: a follow-up study. Clinical Endocrinology 2001 54 547-550. (https://doi.org/10.1046/j.1365-2265.2001.01252.x)

4 Rodanaki M, Lodefalk M, Forssell K, Arvidsson CG, Forssberg M \& Åman J. The incidence of childhood thyrotoxicosis is increasing in both girls and boys in Sweden. Hormone Research in Paediatrics 201991 195-202. (https://doi.org/10.1159/000500265)

5 Flynn RW, MacDonald TM, Morris AD, Jung RT \& Leese GP. The thyroid epidemiology, audit, and research study: thyroid dysfunction in the general population. Journal of Clinical Endocrinology and Metabolism 200489 3879-3884. (https://doi.org/10.1210/jc.2003 032089)

6 Rivkees SA. Pediatric Graves' disease: management in the postpropylthiouracil Era. International Journal of Pediatric Endocrinology 20142014 10. (https://doi.org/10.1186/1687-9856-2014-10) 
7 Simon M, Rigou A, Le Moal J, Zeghnoun A, Le Tertre A, De CrouyChanel P, Kaguelidou F \& Leger J. Epidemiology of childhood hyperthyroidism in France: a nationwide population-based study. Journal of Clinical Endocrinology and Metabolism 2018103 2980-2987. (https://doi.org/10.1210/jc.2018-00273)

8 Mikoś H, Mikoś M, Obara-Moszyńska M \& Niedziela M. The role of the immune system and cytokines involved in the pathogenesis of autoimmune thyroid disease (AITD). Endokrynologia Polska 201465 150-155. (https://doi.org/10.5603/EP.2014.0021)

9 Kuś A, Radziszewski M, Glina A, Szymański K, Jurecka-Lubieniecka B, Pawlak-Adamska E, Kula D, Wawrusiewicz-Kurylonek N, Kuś J, Miśkiewicz P, et al. Paediatric-onset and adult-onset Graves' disease share multiple genetic risk factors. Clinical Endocrinology 201990 320-327. (https://doi.org/10.1111/cen.13887)

10 Bartalena L, Baldeschi L, Boboridis K, Eckstein A, Kahaly GJ, Marcocci C, Perros P, Salvi M, Wiersinga WM \& European Group on Graves. 'The 2016 European Thyroid Association/European Group on Graves' Orbitopathy Guidelines for the Management of Graves' Orbitopathy. European Thyroid Journal 20165 9-26. (https://doi. org/10.1159/000443828)

11 Niedziela M, Breborowicz D, Trejster E \& Korman E. Hot nodules in children and adolescents in western Poland from 1996 to 2000: clinical analysis of 31 patients. Journal of Pediatric Endocrinology and Metabolism 200215 823-830. (https://doi.org/10.1515/jpem.2002.15.6.823)

12 Eszlinger M, Niedziela M, Typlt E, Jaeschke H, Huth S, Schaarschmidt J, Aigner T, Trejster E, Krohn K, Bösenberg E, et al. Somatic mutations in 33 benign and malignant hot thyroid nodules in children and adolescents. Molecular and Cellular Endocrinology 2014393 39-45. (https://doi.org/10.1016/j.mce.2014.05.023)

13 Calebiro D, Grassi ES, Eszlinger M, Ronchi CL, Godbole A, Bathon K, Guizzardi F, de Filippis T, Krohn K, Jaeschke H, et al. Recurrent EZH1 mutations are a second hit in autonomous thyroid adenomas. Journal of Clinical Investigation $20161263383-3388$. (https://doi.org/10.1172/ JCI84894)

14 Paschke R, Niedziela M, Vaidya B, Persani L, Rapoport B \& Leclere J. 2012 European Thyroid Association guidelines for the management of familial and persistent sporadic non-autoimmune hyperthyroidism caused by thyroid-stimulating hormone receptor germline mutations. European Thyroid Journal 20121 142-147. (https://doi. org/10.1159/000342982)

15 Siuda K, Kolesińska Z \& Niedziela M. Amiodaron a funkcja tarczycy [amiodarone and thyroid function]. Kardiologia Polska 201169 493-498.

16 Gesing J, Hoppmann J, Gebauer R, Pfäffle R, Bertsche A \& Kiess W. Diagnosis and clinical course of three adolescents with amiodaroneinduced hyperthyroidism. Pediatric Cardiology 201839 1707-1716. (https://doi.org/10.1007/s00246-018-1981-x)

17 Pappa T \& Refetoff S. Human genetics of thyroid hormone receptor beta: resistance to thyroid hormone beta (RTH $\beta)$. Methods in Molecular Biology 20181801 225-240. (https://doi.org/10.1007/978-1-4939-79028_18)

18 Esen İ, Bayramoğlu E, Yıldız M, Aydın M, Karakılıç Özturhan E, Aycan Z, Bolu S, Önal H, Kör Y, Ökdemir D, et al. Management of thyrotoxicosis in children and adolescents: a Turkish multi-center experience. Journal of Clinical Research in Pediatric Endocrinology 2019 11 164-172. (https://doi.org/10.4274/jcrpe.galenos.2018.2018.0210)

19 Rivkees SA \& Mattison DR. Ending propylthiouracil-induced liver failure in children. New England Journal of Medicine $20093 \mathbf{6 0}$ 1574-1575. (https://doi.org/10.1056/NEJMc0809750)

20 Kahaly GJ, Bartalena L, Hegedüs L, Leenhardt L, Poppe K \& Pearce SH. European Thyroid Association guideline for the management of graves' hyperthyroidism. European Thyroid Journal 20187 167-186. (https://doi.org/10.1159/000490384)

21 Rivkees SA. Controversies in the management of Graves' disease in children. Journal of Endocrinological Investigation 201639 1247-1257. (https://doi.org/10.1007/s40618-016-0477-x)
22 Rabon S, Burton AM \& White PC. Graves' disease in children: longterm outcomes of medical therapy. Clinical Endocrinology 201685 632-635. (https://doi.org/10.1111/cen.13099)

23 Wood CL, Cole M, Donaldson M, Dunger DB, Wood R, Morrison N, Matthews JNS, Pearce SHS \& Cheetham TD. Randomised trial of block and replace vs dose titration thionamide in young people with thyrotoxicosis. European Journal of Endocrinology 2020183 637-645. (https://doi.org/10.1530/EJE-20-0617)

24 Vigone MC, Peroni E, Di Frenna M, Mora S, Barera G \& Weber G. 'Block-and-replace' treatment in Graves' disease: experience in a cohort of pediatric patients. Journal of Endocrinological Investigation 202043 595-600. (https://doi.org/10.1007/s40618-019-01144-0)

25 Léger J, Gelwane G, Kaguelidou F, Benmerad M, Alberti C \& French Childhood Graves' Disease Study Group. Positive impact of longterm antithyroid drug treatment on the outcome of children with Graves' disease: national long-term cohort study. Journal of Clinical Endocrinology and Metabolism 201297 110-119. (https://doi. org/10.1210/jc.2011-1944)

26 Léger J \& Carel JC. MANAGEMENT OF ENDOCRINE DISEASE: Arguments for the prolonged use of antithyroid drugs in children with Graves' disease. European Journal of Endocrinology 2017177 R59-R67. (https://doi.org/10.1530/EJE-16-0938)

27 Chiang YT, Ting WH, Huang CY, Huang SK, Chan CI, Cheng BW, Lin CH, Wu YL, Hung CM, Li HJ, et al. Long-term outcomes of Graves' disease in children treated with anti-thyroid drugs. Pediatrics and Neonatology 2020 61 311-317. (https://doi.org/10.1016/j.pedneo.2019.12.009)

28 Harvengt J, Boizeau P, Chevenne D, Zenaty D, Paulsen A, Simon D, Guilmin Crepon S, Alberti C, Carel JC \& Léger J. Triiodothyroninepredominant Graves' disease in childhood: detection and therapeutic implications. European Journal of Endocrinology 2015172 715-723. (https://doi.org/10.1530/EJE-14-0959)

29 van Lieshout JM, Mooij CF, van Trotsenburg ASP \& ZwavelingSoonawala N. Methimazole-induced remission rates in pediatric Graves' disease: a systematic review. European Journal of Endocrinology 2021185 219-229. (https://doi.org/10.1530/EJE-21-0077)

30 MacFarland SP, Bauer AJ, Adzick NS, Surrey LF, Noyes J, Kazahaya K \& Mostoufi-Moab S. Disease burden and outcome in children and young adults with concurrent Graves disease and differentiated thyroid carcinoma. Journal of Clinical Endocrinology and Metabolism 2018103 2918-2925. (https://doi.org/10.1210/jc.2018-00026)

31 Shi HH \& McHenry CR. Coexistent thyroid nodules in patients with Graves' disease: what is the frequency and the risk of malignancy? American Journal of Surgery 2018216 980-984. (https://doi. org/10.1016/j.amjsurg.2018.07.011)

32 Machens A, Elwerr M, Schneider R, Lorenz K \& Dralle H. Disease impacts more than age on operative morbidity in children with Graves' disease after total thyroidectomy. Surgery 2018164 993-997. (https://doi.org/10.1016/j.surg.2018.07.021)

33 Baumgarten HD, Bauer AJ, Isaza A, Mostoufi-Moab S, Kazahaya K \& Adzick NS. Surgical management of pediatric thyroid disease: complication rates after thyroidectomy at the Children's Hospital of Philadelphia high-volume Pediatric Thyroid Center. Journal of Pediatric Surgery 201954 1969-1975. (https://doi.org/10.1016/j. jpedsurg.2019.02.009)

34 Elfenbein DM, Katz M, Schneider DF, Chen H \& Sippel RS. Thyroidectomy for Graves' disease in children: indications and complications. Journal of Pediatric Surgery 201651 1680-1683. (https:// doi.org/10.1016/j.jpedsurg.2016.03.009)

35 Chen Y, Masiakos PT, Gaz RD, Hodin RA, Parangi S, Randolph GW, Sadow PM \& Stephen AE. Pediatric thyroidectomy in a high-volume thyroid surgery center: risk factors for postoperative hypocalcemia. Journal of Pediatric Surgery 201550 1316-1319. (https://doi. org/10.1016/j.jpedsurg.2014.10.056)

36 Dream S, Wang R, Lovell K, Iyer P, Chen H \& Lindeman B. Outpatient thyroidectomy in the pediatric population. American Journal of Surgery 2020219 890-893. (https://doi.org/10.1016/j.amjsurg.2020.03.025)

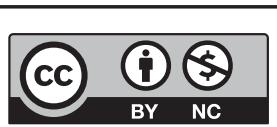

This work is licensed under a Creative Commons Attribution-NonCommercial 4.0 International License. 
37 Read Jr CH, Tansey MJ \& Menda Y. A 36-year retrospective analysis of the efficacy and safety of radioactive iodine in treating young Graves' patients. Journal of Clinical Endocrinology and Metabolism 200489 4229-4233. (https://doi.org/10.1210/jc.2003-031223)

38 Rivkees SA \& Dinauer C. An optimal treatment for pediatric Graves' disease is radioiodine. Journal of Clinical Endocrinology and Metabolism 200792 797-800. (https://doi.org/10.1210/jc.2006-1239)

39 Cury AN, Meira VT, Monte O, Marone M, Scalissi NM, Kochi C, Calliari LE \& Longui CA. Clinical experience with radioactive iodine in the treatment of childhood and adolescent Graves' disease. Endocrine Connections 20132 32-37. (https://doi.org/10.1530/EC-120049)

40 Kaplowitz PB, Jiang J \& Vaidyanathan P. Radioactive iodine therapy for pediatric Graves' disease: a single-center experience over a 10-year period. Journal of Pediatric Endocrinology and Metabolism 202033 383-389. (https://doi.org/10.1515/jpem-2019-0316)

41 Namwongprom S, Dejkhamron P \& Unachak K. Success rate of radioactive iodine treatment for children and adolescent with hyperthyroidism. Journal of Endocrinological Investigation 202144 541-545. (https://doi.org/10.1007/s40618-020-01339-w)

42 Kaplowitz PB \& Vaidyanathan P. Update on pediatric hyperthyroidism. Current Opinion in Endocrinology, Diabetes, and Obesity 202027 70-76. (https://doi.org/10.1097/MED.0000000000000521)

43 Ballal S, Soundararajan R, Singh H, Garg A, Chopra S \& Bal C. Influence of prior carbimazole on the outcome of radioiodine therapy in pediatric and adolescent Graves' disease. Nuclear Medicine Communications 201536 566-572. (https://doi.org/10.1097/ MNM.0000000000000291)

44 Rohrs HJ 3rd, Silverstein JH, Weinstein DA, Amdur RJ \& Haller MJ. Thyroid storm following radioactive iodine (RAI) therapy for pediatric Graves disease. American Journal of Case Reports 201415 212-215. (https://doi.org/10.12659/AJCR.890366)

45 Laurberg P, Wallin G, Tallstedt L, Abraham-Nordling M, Lundell G \& Tørring O. TSH-receptor autoimmunity in Graves' disease after therapy with anti-thyroid drugs, surgery, or radioiodine: a 5-year prospective randomized study. European Journal of Endocrinology 2008 158 69-75. (https://doi.org/10.1530/EJE-07-0450)

46 Watanabe N, Noh JY, Kozaki A, Iwaku K, Sekiya K, Kosuga Y, Matsumoto M, Suzuki M, Yoshihara A, Ohye H, et al. Radioiodineassociated exacerbation of Graves' orbitopathy in the Japanese population: randomized prospective study. Journal of Clinical Endocrinology and Metabolism 2015100 2700-2708. (https://doi. org/10.1210/jc.2014-4542)

47 Mizokami T, Hamada K, Maruta T, Higashi K \& Tajiri J. Long-term outcomes of radioiodine therapy for juvenile Graves disease with emphasis on subsequently detected thyroid nodules: a single institution experience from Japan. Endocrine Practice 202026 729-737. (https://doi.org/10.4158/EP-2019-0468)

48 Cohen RZ, Felner EI, Heiss KF, Wyly JB \& Muir AB. Outcomes analysis of radioactive iodine and total thyroidectomy for pediatric Graves' disease. Journal of Pediatric Endocrinology and Metabolism 201629 319-325. (https://doi.org/10.1515/jpem-2015-0333)

49 Zirilli G, Velletri MR, Porcaro F, Di Giovine G, Maisano P \& La Monica G. Hyperthyroidism in childhood: peculiarities of the different clinical pictures. Acta Bio-Medica: Atenei Parmensis 201586 220-225.

50 Bauer AJ. Approach to the pediatric patient with Graves' disease: when is definitive therapy warranted? Journal of Clinical Endocrinology and Metabolism 201196 580-588. (https://doi.org/10.1210/jc.2010-0898)

51 Rivkees SA. Pediatric Graves' disease: controversies in management. Hormone Research in Paediatrics 201074 305-311. (https://doi. org/10.1159/000320028)
52 De Luca F \& Valenzise M. Controversies in the pharmacological treatment of Graves' disease in children. Expert Review of Clinical Pharmacology 201811 1113-1121. (https://doi.org/10.1080/17512433.20 18.1546576)

53 Niedziela M, Korman E, Breborowicz D, Trejster E, Harasymczuk J, Warzywoda M, Rolski M \& Breborowicz J. A prospective study of thyroid nodular disease in children and adolescents in western Poland from 1996 to 2000 and the incidence of thyroid carcinoma relative to iodine deficiency and the chernobyl disaster. Pediatric Blood and Cancer 200442 84-92. (https://doi.org/10.1002/pbc.10421)

54 Niedziela M \& Korman E. Thyroid carcinoma in a fourteen-yearold boy with Graves disease. Medical and Pediatric Oncology 200238 290-291. (https://doi.org/10.1002/mpo.1330)

55 Shimura K, Shibata H, Mizuno Y, Amano N, Hoshino K, Kuroda T, Kameyama K, Matsuse M, Mitsutake N, Sugino K, et al. Rapid growth and early metastasis of papillary thyroid carcinoma in an adolescent girl with Graves' disease. Hormone Research in Paediatrics 201991 210-215. (https://doi.org/10.1159/000491102)

56 Kojima-Ishii K, Ihara K, Ohkubo K, Matsuo T, Toda N, Yamashita H, Kono S \& Hara T. Thyroid follicular carcinoma in a fourteen-year-old girl with Graves' disease. Clinical Pediatric Endocrinology 201423 59-64. (https://doi.org/10.1297/cpe.23.59)

57 Kovatch KJ, Bauer AJ, Isaacoff EJ, Prickett KK, Adzick NS, Kazahaya K, Sullivan LM \& Mostoufi-Moab S. Pediatric thyroid carcinoma in patients with Graves' disease: the role of ultrasound in selecting patients for definitive therapy. Hormone Research in Paediatrics 201583 408-413. (https://doi.org/10.1159/000381185)

58 Jia Q, Li X, Liu Y, Li L, Kwong JS, Ren K, Jiang Y, Sun X, Tian H \& Li S. Incidental thyroid carcinoma in surgery-treated hyperthyroid patients with Graves' disease: a systematic review and meta-analysis of cohort studies. Cancer Management and Research 201810 1201-1207. (https:// doi.org/10.2147/CMAR.S164210)

59 Niedziela M. Pathogenesis, diagnosis and management of thyroid nodules in children. Endocrine-Related Cancer 200613 427-453. (https://doi.org/10.1677/erc.1.00882)

60 Niedziela M. Thyroid nodules. Best Practice and Research: Clinical Endocrinology and Metabolism 201428 245-277. (https://doi. org/10.1016/j.beem.2013.08.007)

$61 \mathrm{Wu}$ CY, Elner VM \& Kahana A. Severe pediatric thyroid eye disease: surgical case series. Ophthalmic Plastic and Reconstructive Surgery 201733 (3S Supplement 1) S186-S188. (https://doi.org/10.1097/ IOP.0000000000000585)

62 Boelaert K, Visser WE, Taylor PN, Moran C, Léger J \& Persani L. ENDOCRINOLOGY IN THE TIME OF COVID-19: Management of hyperthyroidism and hypothyroidism. European Journal of Endocrinology 2020183 G33-G39. (https://doi.org/10.1530/EJE-20-0445)

63 Glaser NS, Styne DM \& Organization of Pediatric Endocrinologists of Northern California Collaborative Graves' Disease Study Group. Predicting the likelihood of remission in children with Graves' disease: a prospective, multicenter study. Pediatrics 2008121 e481-e488. (https://doi.org/10.1542/peds.2007-1535)

64 Kaguelidou F, Alberti C, Castanet M, Guitteny MA, Czernichow P, Léger J \& French Childhood Graves' Disease Study Group. Predictors of autoimmune hyperthyroidism relapse in children after discontinuation of antithyroid drug treatment. Journal of Clinical Endocrinology and Metabolism 200893 3817-3826. (https://doi.org/10.1210/jc.2008-0842)

65 Jevalikar G, Solis J \& Zacharin M. Long-term outcomes of pediatric Graves' disease. Journal of Pediatric Endocrinology and Metabolism 2014 27 1131-1136. (https://doi.org/10.1515/jpem-2013-0342)

66 Lane LC, Cheetham TD, Perros P \& Pearce SHS. New therapeutic horizons for Graves' hyperthyroidism. Endocrine Reviews 202041 873-884. (https://doi.org/10.1210/endrev/bnaa022)

Received in final form 26 August 2021

Accepted 30 September 2021

Accepted Manuscript published online 1 October 2021 https://ec.bioscientifica.com https://doi.org/10.1530/EC-21-0191 (c) 2021 The authors Published by Bioscientifica Ltd
This work is licensed under a Creative Commons Attribution-NonCommercial 4.0 International License. 